\title{
PERIODIC ORBITS BIFURCATING FROM A NON-ISOLATED ZERO-HOPF EQUILIBRIUM OF THREE-DIMENSIONAL DIFFERENTIAL SYSTEMS REVISITED
}

\author{
Murilo R. Cândido \\ Departament de Matemàtiques, Universitat Autònoma de Barcelona, Bellaterra \\ Barcelona, 08193, Spain \\ candidomr@mat.uab.cat \\ Jaume Llibre \\ Departament de Matemàtiques, Universitat Autònoma de Barcelona, Bellaterra \\ Barcelona, 08193, Spain \\ jllibre@mat.uab.cat
}

Received (to be inserted by publisher)

\begin{abstract}
In this paper we study the periodic solutions bifurcating from a non-isolated zero-Hopf equilibrium in a polynomial differential system of degree two in $\mathbb{R}^{3}$. More specifically, we use recent results of averaging theory to improve the conditions for the existence of one or two periodic solutions bifurcating from such a zero-Hopf equilibrium. This new result is applied for studying the periodic solutions of differential systems in $\mathbb{R}^{3}$ having $n$-scroll chaotic attractors.
\end{abstract}

Keywords: averaging theory, periodic solutions, polynomial differential systems, zero-Hopf bifurcation, zero-Hopf equilibrium.

\section{Introduction and Statement of the Main Result}

In this paper we study the periodic orbits bifurcating from a non-isolated zero-Hopf equilibrium of a three-dimensional autonomous differential system, it means that the differential system has a continuum of equilibria containing a point with a zero eigenvalue and a pair of purely imaginary eigenvalues. The zeroHopf bifurcation is an interesting subject in differential systems and have been studied by Guckenheimer [1981]; Guckenheimer \& Holmes [2013]; Scheurle \& Marsden [1984]; Kuznetsov [2013] and many other authors. Usually the zero-Hopf bifurcation is a two-parameter unfolding of a three-dimensional autonomous differential system with an isolated zero-Hopf equilibrium. It is known that some complicated invariant sets can bifurcate from an isolated zero-Hopf equilibrium, for instance zero-Hopf bifurcation can imply local birth of "chaos", see for instance Scheurle \& Marsden [1984].

Most papers study isolated zero-Hopf equilibrium. One of the few works about non-isolated zero-Hopf equilibrium was done in 2012 by Llibre \& Xiao [2014]. The authors studied the periodic orbits bifurcating 
from the non-isolated zero-Hopf equilibrium located at the origin of the following family of polynomial differential systems of degree two

$$
\begin{aligned}
\frac{d U}{d t} & =\varepsilon \lambda U-\omega V+\sum_{i+j+k=2} a_{i j k}(\varepsilon) U^{i} V^{j} W^{k}, \\
\frac{d V}{d t} & =\omega U+\varepsilon \lambda V+\sum_{i+j+k=2} b_{i j k}(\varepsilon) U^{i} V^{j} W^{k}, \\
\frac{d W}{d t} & =\varepsilon \mu W+\sum_{i+j+k=2} c_{i j k}(\varepsilon) U^{i} V^{j} W^{k},
\end{aligned}
$$

with $\mu=1$ and where the coefficients functions are of the form

$$
\begin{aligned}
& a_{i j k}(\varepsilon)=\sum_{l=0}^{1} a_{i j k l} \varepsilon^{l}+\mathcal{O}\left(\varepsilon^{2}\right), \\
& b_{i j k}(\varepsilon)=\sum_{l=0}^{1} b_{i j k l} \varepsilon^{l}+\mathcal{O}\left(\varepsilon^{2}\right), \\
& c_{i j k}(\varepsilon)=\sum_{l=0}^{1} c_{i j k l} \varepsilon^{l}+\mathcal{O}\left(\varepsilon^{2}\right) .
\end{aligned}
$$

When $\varepsilon=0$, this system has a continuum of equilibria which fill a segment, or a half-straight line. As shown by Llibre \& Xiao [2014, Proposition 2.2 ] this continuum of equilibria will have a (non-isolated) zero-Hopf equilibrium at the origin if and only if system (1) satisfies the following hypothesis

$$
\left(H_{0}\right): a_{0020}=b_{0020}=c_{0020}=0 .
$$

In a small neighbourhood of the origin the authors reduced system (1) to a $2 \pi$-periodic differential system using a kind of cylindrical coordinates and a scaling of the variables. Then second order averaging theory was used for providing explicit conditions for the existence of one or two periodic orbits bifurcating from the non-isolated zero-Hopf equilibrium, see Llibre \& Xiao [2014, Theorem 2.4].

We shall use recent results obtained in the averaging theory to weak the hypotheses of Llibre \& Xiao [2014, Theorem 2.4] improving those results. Mainly their result was obtained assuming the following hypothesis

$$
\left(H_{1}\right): c_{2000}=c_{1100}=c_{0200}=0 .
$$

We extend these results giving sufficient conditions for the existence of $\varepsilon$-families of periodic orbits bifurcating from the origin of system (1) using the weaker hypothesis

$$
\left(H_{1}^{\prime}\right): c_{2000}=-c_{0200} .
$$

This will be our main result, see Theorem 1. In section 2 we formulate the averaging theorem (see Theorem 2) used for proving our main result. We use averaging theory in this paper, although the same kind of study could be done by Melnikov method. In particular, the Melnikov functions recently developed by Tian \& Han [2017] could also be applied here. In section 3 we shall apply Theorem 1 to study three polynomial differential systems of degree two that cannot be studied using Llibre \& Xiao [2014, Theorem 2.4]. More precisely these three polynomial differential systems are system (11) proposed by Li [2008], system (12) provided by Pan et al. [2010] and system (10) proposed by Elhadj \& Sprott [2013]. For certain coefficients values all these systems present $n$-scroll chaotic attractors. They also have non-isolated zeroHopf equilibria, and we shall use Theorem 1 to give sufficient conditions for the existence of periodic orbits for these systems.

Our main result is the following. 
Theorem 1. Assume that $\left(H_{0}\right)$ holds. Then system (1) has a non-isolated zero-Hopf equilibrium at the origin when $\varepsilon=0$. Furthermore if the assumption $\left(H_{1}^{\prime}\right)$ holds, there is $\varepsilon^{*}$ such that $0<\varepsilon^{*} \ll 1$ and for any $\varepsilon$ and $0<\varepsilon<\varepsilon^{*}$, the following statements hold.

(i) System (1) has one family of periodic orbits bifurcating from the origin if one of the following conditions holds:

(a) $a_{1010}+b_{0110}=0, \lambda B_{1}<0$ and $\lambda\left(B_{1}+B_{4}\right) \neq B_{1} \mu$,

(b) $a_{1010}+b_{0110} \neq 0, \lambda \mu=0$ and $Q_{1} Q_{2}<0$,

(c) $a_{1010}+b_{0110} \neq 0, Q_{1}=0$ and $Q_{2} \lambda \mu<0$,

(d) $a_{1010}+b_{0110} \neq 0, Q_{2}=0, \mu \lambda>0$ and $Q_{1}<0$,

(e) $a_{1010}+b_{0110} \neq 0,3 Q_{2}^{2}-4 Q_{1} \lambda \mu \neq 0, Q_{2} \neq 0, \mu \lambda>0$ and $Q_{1}<0$,

(f) $a_{1010}+b_{0110} \neq 0,3 Q_{2}^{2}-4 Q_{1} \lambda \mu \neq 0, Q_{2} \neq 0, \mu \lambda<0$ and $Q_{1}>0$.

(ii) System (1) has two families of periodic orbits bifurcating from the origin if one of the following condition holds:

(g) $a_{1010}+b_{0110} \neq 0,3 Q_{2}^{2}-4 Q_{1} \lambda \mu \neq 0, Q_{2}<0, \lambda \mu>0$ and $0<Q_{1}<3 Q_{2}^{2} /(4 \lambda \mu)$,

(h) $a_{1010}+b_{0110} \neq 0,3 Q_{2}^{2}-4 Q_{1} \lambda \mu \neq 0, Q_{2}>0, \lambda \mu<0$ and $3 Q_{2}^{2} /(4 \lambda \mu)<Q_{1}<0$,

where

$$
\begin{aligned}
B_{1} & =4 a_{2000}\left(b_{2000}+2 c_{0110}\right)-2 a_{0200}\left(a_{1100}+2 b_{0200}-4 c_{0110}\right) \\
& -c_{1100}\left(a_{1010}+11 b_{0110}-8 c_{0020}\right)-10 c_{2000}\left(a_{0110}+b_{1010}\right) \\
& -2 a_{1100} a_{2000}+2\left(b_{0200}+b_{2000}\right)\left(b_{1100}-4 c_{1010}\right) \\
B_{2} & =2 a_{0200}\left(181 a_{2000}+67 b_{1100}-80 c_{1010}\right)-2 a_{1100}\left(61 b_{0200}\right. \\
& \left.+67 b_{2000}-8 c_{0110}\right)-16 c_{1010}\left(8 a_{2000}+b_{1100}\right)+72 c_{1100}\left(a_{0110}\right. \\
& \left.+b_{1010}\right)+122 a_{2000} b_{1100}+235 a_{0200}^{2}+5 a_{1100}^{2}+123 a_{2000}^{2}+32\left(4 b_{0200}\right. \\
& \left.+5 b_{2000}\right) c_{0110}-123 b_{0200}^{2}-5 b_{1100}^{2}-235 b_{2000}^{2}-362 b_{0200} b_{2000}, \\
B_{3} & =a_{0200}\left(a_{1100}-233 b_{0200}-47 b_{2000}+112 c_{0110}\right)+a_{1100}\left(7 a_{2000}\right. \\
& \left.+b_{1100}-8 c_{1010}\right)-303 a_{2000} b_{0200}-137 a_{2000} b_{2000}+128 a_{2000} c_{0110} \\
& -8 b_{1100} c_{0110}+16\left(2 b_{0200}+b_{2000}\right) c_{1010}+36 c_{1100}\left(c_{0020}-2 b_{0110}\right) \\
& +55 b_{1100} b_{0200}+49 b_{1100} b_{2000}, \\
B_{4} & =4 a_{2000}\left(b_{2000}+2 c_{0110}\right)-2 a_{0200}\left(a_{1100}+2 b_{0200}-4 c_{0110}\right) \\
& -c_{1100}\left(a_{1010}+11 b_{0110}-8 c_{0020}\right)-10 c_{2000}\left(a_{0110}+b_{1010}\right) \\
& -2 a_{1100} a_{2000}+2\left(b_{0200}+b_{2000}\right)\left(b_{1100}-4 c_{1010}\right), \\
Q_{1} & =\left(a_{1010}+b_{0110}\right)\left(c_{2000}\left(48 c_{2000}\left(a_{1010}+b_{0110}\right)+B_{3}\right)-B_{2} c_{1100}\right) \\
& +3 B_{1}\left(B_{1}+B_{4}\right), \\
Q_{2} & =-2\left(a_{1010}+b_{0110}\right)\left(c_{1100}(\mu-2 \lambda)+2 \omega\left(c_{0,2,0,1}+c_{2,0,0,1}\right)\right) \\
& +B_{1}(\lambda+\mu)+B_{4} \lambda .
\end{aligned}
$$

\section{Averaging Theory and Proof of Theorem 1}

To find the periodic orbits bifurcating from a non-isolated zero-Hopf equibilbrium of the differential system (1) we use averaging theory. The averaging theory has a long history and for a modern exposition of this topic the reader is addressed to Murdock et al. [2007].

We are interested in the formulation of the averaging theory for systems with non-trivial unperturbed part, i.e., we consider the differential system 


$$
\dot{x}(t)=F_{0}(t, x)+\varepsilon F_{1}(t, x)+\varepsilon^{2} F_{2}(t, x)+\varepsilon^{3} R(t, x, \varepsilon),
$$

where $F_{i}: \mathbb{R} \times D \rightarrow \mathbb{R}^{n}$ for $i=1,2$, and $R: \mathbb{R} \times D \times\left(-\varepsilon_{0}, \varepsilon_{0}\right) \rightarrow \mathbb{R}^{n}$ are continuous functions, $T$-periodic in the first variable and $D$ is an open subset of $\mathbb{R}^{n}$. There are several recent works developing and improving the averaging theory for this kind of systems, see for instance [Cândido et al., 2016; Coll et al., 2012; Llibre et al., 2014; Giné et al., 2016]. For the convenience of the reader we present here the second order averaging theory for system $(3)$. Let $\Phi(\cdot, z):\left[0, t_{z}\right] \rightarrow \mathbb{R}^{n}$ be the solution of the unperturbed system, $\dot{x}(t)=F_{0}(t, x)$, such that $\Phi(0, z)=z$. For $i=1,2$ we define the following averaged functions $g_{i}: D \rightarrow \mathbb{R}^{n}$ of order $i$ as

$$
g_{i}(z)=M(T, z)^{-1} \frac{y_{i}(T, z)}{i !},
$$

where

$$
\begin{aligned}
y_{1}(t, z)= & M(t, z) \int_{0}^{t} M(\tau, z)^{-1} F_{1}(\tau, \Phi(\tau, z)) d \tau \\
y_{2}(t, z)= & M(t, z) \int_{0}^{t} M(\tau, z)^{-1}\left[2 F_{2}(\tau, \Phi(\tau, z))+\frac{d F_{1}}{d z}(\tau, \Phi(\tau, z)) \cdot y_{1}(\tau, z)\right. \\
& \left.+\frac{d^{2} F_{0}}{d x^{2}}(\tau, \Phi(\tau, z)) y_{1}(\tau, z) d \tau\right]
\end{aligned}
$$

and $M(t, z)$ is the fundamental matrix of the variational differential equation of the unperturbed system along the solution $\Phi(t, z)$, such that $M(0, z)$ is the identity matrix. The next theorem provides the second order averaging theory, for a proof see Llibre et al. [2014].

Theorem 2. Assume the following conditions.

(i) There exists an open subset $W$ of $D$ such that for any $z \in \bar{W}, \Phi(t, z)$ is T-periodic in the variable $t$.

(ii) Assume that $g_{1}(z) \equiv 0$, and that there exists $a^{*} \in W$ with $g_{2}\left(a^{*}\right)=0$ and $\operatorname{det}\left(D g_{2}\left(a^{*}\right)\right) \neq 0$.

Then for $|\varepsilon| \neq 0$ sufficiently small there exists a T-periodic solution $x(t, z(\varepsilon), \varepsilon)$ of system (3) such that $\left|z(\varepsilon)-a^{*}\right|=\mathcal{O}(\varepsilon)$.

Now we shall use Theorem 2 for proving our main result.

Proof. [Proof of Theorem 1] Assuming hypotheses $\left(H_{0}\right)$ and $\left(H_{1}^{\prime}\right)$, we start by writing system (1) into the normal form for applying the averaging Theorem 2. We use the change of coordinates $U=R \cos \theta$, $V=R \sin \theta$ and $W=R Z$. Then we scale the system taking $R=\sqrt{\varepsilon} r$ and $Z=\sqrt{\varepsilon} z$ with $\varepsilon>0$ a small parameter. These are exactly the same steps used to obtain the equation (2.8) of [Llibre \& Xiao, 2014]. Taking $\theta$ as the new independent variable we obtain the following differential system

$$
\left(\frac{d r}{d \theta}, \frac{d z}{d \theta}\right)=F_{0}(\theta, r, z)+\sqrt{\varepsilon} F_{1}(\theta, r, z)+\varepsilon F_{2}(\theta, r, z)+\mathcal{O}\left(\varepsilon^{\frac{3}{2}}\right)
$$

where $F_{i}(\theta, r, z)=\left(F_{i}^{1}(\theta, r, z), F_{i}^{2}(\theta, r, z)\right)$ are $2 \pi$-periodic in the variable $\theta$ for $i=0,1,2$, and are given by

$$
\begin{aligned}
F_{0}^{1}(\theta, r, z)= & 0 \\
F_{0}^{2}(\theta, r, z)= & \frac{r}{\omega}\left(c_{0200} \sin ^{2} \theta+\cos \theta\left(c_{1100} \sin \theta+c_{2000} \cos \theta\right)\right), \\
F_{1}^{1}(\theta, r, z)= & \frac{r^{2}}{\omega}\left(a_{2000} \cos ^{3} \theta+a_{1100} \sin \theta \cos ^{2} \theta+a_{0200} \sin ^{2} \theta \cos \theta\right. \\
& \left.+b_{0200} \sin ^{3} \theta+b_{2000} \sin \theta \cos ^{2} \theta+b_{1100} \sin ^{2} \theta \cos \theta\right),
\end{aligned}
$$




$$
\begin{aligned}
& F_{1}^{2}(\theta, r, z)=\frac{r}{\omega}\left(r ( c _ { 2 0 0 0 } \operatorname { c o s } ( 2 \theta ) + c _ { 1 1 0 0 } \operatorname { s i n } ( \theta ) \operatorname { c o s } ( \theta ) ) \left(a_{0200} \sin ^{3}(\theta)\right.\right. \\
& -\cos (\theta)\left(-a_{1100} \sin ^{2}(\theta)-a_{2000} \sin (\theta) \cos (\theta)\right. \\
& \left.\left.+b_{0200} \sin ^{2}(\theta)+b_{2000} \cos ^{2}(\theta)+b_{1100} \sin (\theta) \cos (\theta)\right)\right) \\
& -\omega z\left(a_{2000} \cos ^{3}(\theta)+a_{1100} \sin (\theta) \cos ^{2}(\theta)+a_{0200} \sin ^{2}(\theta) \cos (\theta)\right. \\
& +b_{0200} \sin ^{3}(\theta)+b_{2000} \sin (\theta) \cos ^{2}(\theta)+b_{1100} \sin ^{2}(\theta) \cos (\theta) \\
& \left.\left.-c_{0110} \sin (\theta)-c_{1010} \cos (\theta)\right)\right) \\
& \left.\left(c_{0200} \sin ^{2} \theta+\cos \theta\left(c_{1100} \sin \theta+c_{2000} \cos \theta\right)\right)\right) \text {, } \\
& F_{2}^{1}(\theta, r, z)=\frac{r}{\omega}\left(\lambda+r z\left(a_{1010} \cos ^{2} \theta+a_{0110} \sin \theta \cos \theta+\sin \theta\right.\right. \\
& \left.\left(b_{0110} \sin \theta+b_{1010} \cos \theta\right)\right)+\frac{r^{2}}{\omega}\left(\operatorname { s i n } \theta \operatorname { c o s } \theta \left(\operatorname { s i n } \theta \left(a_{1100}\right.\right.\right. \\
& \left.\left.\left.-b_{0200}\right)+\cos \theta\left(a_{2000}-b_{1100}\right)\right)+a_{0200} \sin ^{3} \theta-b_{2000} \cos ^{3} \theta\right) \\
& \left(a_{2000} \cos ^{3} \theta+a_{1100} \sin \theta \cos ^{2} \theta+a_{0200} \sin ^{2} \theta \cos \theta\right. \\
& \left.\left.+b_{0200} \sin ^{3} \theta+b_{2000} \sin \theta \cos ^{2} \theta+b_{1100} \sin ^{2} \theta \cos \theta\right)\right) \text {, } \\
& F_{2}^{2}(\theta, r, z)=\frac{z(\mu-\lambda)}{\omega}+\frac{r^{3}}{\omega^{2}}\left(\operatorname { s i n } \theta \operatorname { c o s } \theta \left(\sin \theta\left(a_{1100}-b_{0200}\right)+\cos \theta\right.\right. \\
& \left.\left.\left(a_{2000}-b_{1100}\right)\right)+a_{0200} \sin ^{3} \theta-b_{2000} \cos ^{3} \theta\right)^{2}\left(c_{2000} \cos (2 \theta)\right. \\
& \left.+c_{1100} \sin \theta \cos \theta\right)+\frac{r^{2} z}{\omega^{2}}\left(\left(\operatorname { s i n } \theta \operatorname { c o s } \theta \left(\sin \theta\left(b_{0200}-a_{1100}\right)\right.\right.\right. \\
& \left.\left.+\cos \theta\left(b_{1100}-a_{2000}\right)\right)-a_{0200} \sin ^{3} \theta+b_{2000} \cos ^{3} \theta\right) \\
& \left(a_{2000} \cos ^{3} \theta+a_{1100} \sin \theta \cos ^{2} \theta+a_{0200} \sin ^{2} \theta \cos \theta\right. \\
& +b_{0200} \sin ^{3} \theta+b_{2000} \sin \theta \cos ^{2} \theta+b_{1100} \sin ^{2} \theta \cos \theta-c_{0110} \sin \theta \\
& \left.-c_{1010} \cos \theta\right)+a_{0110} \sin ^{2} \theta\left(c_{2000} \cos (2 \theta)+c_{1100} \sin \theta \cos \theta\right) \\
& +a_{1010} \sin \theta \cos \theta\left(c_{2000} \cos (2 \theta)+c_{1100} \sin \theta \cos \theta\right) \\
& -b_{1010} \cos ^{2} \theta\left(c_{2000} \cos (2 \theta)+c_{1100} \sin \theta \cos \theta\right) \\
& \left.-b_{0110} \sin \theta \cos \theta\left(c_{2000} \cos (2 \theta)+c_{1100} \sin \theta \cos \theta\right)\right) \\
& -\frac{r z^{2}}{\omega}\left(\left(a_{1010} \cos ^{2} \theta+a_{0110} \sin \theta \cos \theta+\sin \theta\left(b_{0110} \sin \theta\right.\right.\right. \\
& \left.\left.\left.+b_{1010} \cos \theta\right)\right)+\frac{c_{0201} \sin ^{2} \theta+\cos \theta\left(c_{1101} \sin \theta+c_{2001} \cos \theta\right)}{\omega}\right) .
\end{aligned}
$$

Consider the unperturbed system corresponding to (5), i. e.

$$
\begin{aligned}
& \frac{d r}{d \theta}=0, \\
& \frac{d z}{d \theta}=\frac{r}{\omega}\left(c_{0200} \sin ^{2} \theta+\cos \theta\left(c_{1100} \sin \theta+c_{2000} \cos \theta\right)\right) .
\end{aligned}
$$

Taking $(\bar{r}, \bar{z}) \in \mathbb{R}^{+} \times \mathbb{R}$ as initial conditions, the unperturbed system has the solution

$$
\Phi(\theta, \bar{r}, \bar{z})=\left(\bar{r}, \frac{\bar{r} c_{1100} \sin ^{2} \theta+\bar{r} c_{2000} \sin (2 \theta)+2 \omega \bar{z}}{2 \omega}\right) .
$$

Thus the solution $\phi(\theta, \bar{r}, \bar{z})$ is $2 \pi$-periodic for all $(\bar{r}, \bar{z}) \in \mathbb{R}^{+} \times \mathbb{R}$, then system (5) satisfies the hypotheses $(i)$ of Theorem 2. Furthermore, the fundamental matrix associated to the variational equation of the solution 
$\phi(\theta, \bar{r}, \bar{z})$ is

$$
M(\theta)=\left(\begin{array}{cc}
1 & 0 \\
\frac{c_{1100}-c_{1100} \cos ^{2} \theta+c_{2000} \sin (2 \theta)}{2 \omega} & 1
\end{array}\right) .
$$

Thus using (4) the averaged functions of system $(5)$ are $g_{1}(\bar{r}, \bar{z})=(0,0)$ and

$$
\begin{gathered}
g_{2}(\bar{r}, \bar{z})=\left(\frac{2 \pi \bar{r}^{2} \bar{z}\left(a_{1010}+b_{0110}\right)}{\omega}+\frac{\pi B_{1} \bar{r}^{3}}{4 \omega^{2}}+\frac{4 \pi \lambda \bar{r}}{\omega}, \frac{\pi \bar{r}^{3}}{96 \omega}\left(B_{2} c_{1100}\right.\right. \\
\left.-B_{3} c_{2000}-48 c_{2000}^{2}\left(a_{1010}+b_{0110}\right)\right)-\bar{r}\left(\frac{2 \pi \bar{z}^{2}\left(a_{1010}+b_{0110}\right)}{\omega}\right. \\
\left.\left.-\frac{\pi\left((\mu-2 \lambda) c_{1100}+2 \omega\left(c_{0201}+c_{2001}\right)\right)}{\omega^{2}}\right)+\frac{\pi B_{4} \bar{r}^{2} \bar{z}}{4 \omega^{2}}+\frac{4 \pi \bar{z}(\mu-\lambda)}{\omega}\right) .
\end{gathered}
$$

Now we have to find the simple zeros of the system

$$
g_{2}(\bar{r}, \bar{z})=(0,0) .
$$

We are interest only in the solutions $\left(r_{0}, z_{0}\right) \in \mathbb{R}^{2}$ such that $g_{2}\left(r_{0}, z_{0}\right)=0$ and $r_{0}>0$. We divide the study of these solutions in the following cases:

Case 1: $a_{1010}+b_{0110}=0$. If $\lambda B_{1}<0$ and $\lambda\left(B_{1}+B_{4}\right) \neq B_{1} \mu$, then system (7) has the solution

$$
\begin{aligned}
\left(r_{0}, z_{0}\right) & =\left(4 \sqrt{\frac{-\lambda \omega}{B_{1}}}, \frac{r_{0}}{6 \omega\left(B_{1} \mu-\lambda\left(B_{1}+B_{4}\right)\right)}\left(c_{1100}\left(6 B_{1} \mu-\lambda\left(12 B_{1}+B_{3}\right)\right)\right.\right. \\
& \left.\left.+12 B_{1} \omega c_{0201}+12 B_{1} \omega c_{2001}+B_{2} \lambda c_{2000}\right)\right) .
\end{aligned}
$$

Furthermore we have

$$
\operatorname{det}\left(D g_{2}\left(r_{0}, z_{0}\right)\right)=\frac{32 \pi^{2} \lambda\left(\left(B_{1}+B_{4}\right) \lambda-\mu B_{1}\right)}{\omega^{2} B_{1}} .
$$

Consequently $\left(r_{0}, z_{0}\right)$ is a simple zero of system $(7)$.

Case $2: a_{1010}+b_{0110} \neq 0$. Solving the first equation of system (7) with respect to $\bar{z}$ and $\bar{r}>0$ we have that $\bar{z}=-\left(B_{1} \bar{r}^{2}+16 \lambda \omega\right) /\left(8 \bar{r} \omega\left(a_{1010}+b_{0110}\right)\right)$. Eliminating $\bar{z}$ in the system (7) we obtain the polynomial,

$$
P(r)=Q_{1} \bar{r}^{4}+48 Q_{2} \bar{r}^{2} \omega+768 \lambda \mu \omega^{2} .
$$

The bi-quadratic polynomial (8) may have one or two real positive roots. Thus we use its discriminant

$$
\text { D : } 7247757312 Q_{1} \lambda \mu\left(3 Q_{2}^{2}-4 Q_{1} \lambda \mu\right)^{2} \omega^{6},
$$

to study such roots and then verify when them provide simple zeros of system (7). The discriminant vanishes if $Q_{1} \lambda \mu=0$ or $3 Q_{2}^{2}-4 Q_{1} \lambda \mu=0$, in this case one can verify the following subcases

Subcase 2.1: If $\lambda \mu=0$ and $Q_{1} Q_{2}<0$, system (7) has the zero

$$
\left(r_{1}, z_{1}\right)=\left(4 \sqrt{\frac{-3 \omega Q_{2}}{Q_{1}}}, \frac{B_{1} r_{1}^{2}}{8 \omega r_{1}\left(a_{1010}+b_{0110}\right)}\right) .
$$

It is a simple zero because $\operatorname{det}\left(D g_{2}\left(r_{1}, z_{1}\right)\right)=\frac{96 \pi^{2} Q_{2}^{2}}{Q_{1} \omega^{2}} \neq 0$.

Subcase 2.2: If $Q_{1}=0$ and $Q_{2} \lambda \mu<0$, system (7) has the zero

$$
\left(r_{2}, z_{2}\right)=\left(4 \sqrt{\frac{-\lambda \mu \omega}{Q_{2}}}, \frac{B_{1} r_{2}^{2}+16 \lambda \omega}{8 \omega r_{2}\left(a_{1010}+b_{0110}\right)}\right) .
$$


It is also a simple zero since $\operatorname{det}\left(D g_{2}\left(r_{1}, z_{1}\right)\right)=-\frac{32 \pi^{2} \lambda \mu}{\omega^{2}} \neq 0$.

Subcase 2.3: If $3 Q_{2}^{2}-4 Q_{1} \lambda \mu=0$, system (7) has no simple zeros.

When the discriminant (9) is non-zero the polynomial (8) has 4 distinct solutions. These solutions may present zero, one or two positive real values for $\bar{r}$. Then assuming $Q_{1} \lambda \mu\left(3 Q_{2}^{2}-4 Q_{1} \lambda \mu\right) \neq 0$ we have the following subcases.

Subcase 2.4: If $Q_{2}<0, \lambda \mu>0$ and $0<Q_{1}<3 Q_{2}^{2} /(4 \lambda \mu)$; or $Q_{2}>0, \lambda \mu<0$ and $Q_{2}^{2} /(4 \lambda \mu)<Q_{1}<0$ system (7) has two zeros

$$
\begin{aligned}
& \left(r_{3}, z_{3}\right)=\left(2 \sqrt{\frac{-\omega\left(6 Q_{2}+2 \sqrt{9 Q_{2}^{2}-12 Q_{1} \lambda \mu}\right)}{Q_{1}}}, \frac{B_{1} r_{3}^{2}+16 \lambda \omega}{8 \omega r_{3}\left(a_{1010}+b_{0110}\right)}\right), \\
& \left(r_{4}, z_{4}\right)=\left(2 \sqrt{\frac{\omega\left(6 Q_{2}-2 \sqrt{9 Q_{2}^{2}-12 Q_{1} \lambda \mu}\right)}{Q_{1}}}, \frac{B_{1} r_{4}^{2}+16 \lambda \omega}{8 \omega r_{4}\left(a_{1010}+b_{0110}\right)}\right) .
\end{aligned}
$$

Furthermore for $i=3,4$ the Jacobian determinant of system (7) is

$$
\operatorname{det}\left(D g_{2}\left(r_{i}, z_{i}\right)\right)=-\frac{64 \pi^{2} \lambda \mu}{\omega^{2}}-\frac{2 \pi^{2} Q_{2} r_{i}^{2}}{\omega^{3}} \neq 0
$$

consequently these zeros are simple.

Subcase 2.5: If $Q_{2}=0, \mu \lambda>0$ and $Q_{1}<0$ system (7) has the zero

$$
\left(r_{5}, z_{5}\right)=\left(2 \sqrt{\frac{-\omega\left(2 \sqrt{-12 Q_{1} \lambda \mu}\right)}{Q_{1}}}, \frac{B_{1} r_{5}^{2}+16 \lambda \omega}{8 \omega r_{5}\left(a_{1010}+b_{0110}\right)}\right),
$$

and its Jacobian determinant is $\operatorname{det}\left(D g_{2}\left(r_{i}, z_{i}\right)\right)=-\frac{64 \pi^{2} \lambda \mu}{\omega^{2}} \neq 0$.

Subcase 2.6: If $Q_{2} \neq 0, \mu \lambda>0$ and $Q_{1}<0$ system (7) has the simple zero

$$
\left(r_{6}, z_{6}\right)=\left(2 \sqrt{\frac{\omega\left(6 Q_{2}-2 \sqrt{9 Q_{2}^{2}-12 Q_{1} \lambda \mu}\right)}{Q_{1}}}, \frac{B_{1} r_{6}^{2}+16 \lambda \omega}{8 \omega r_{6}\left(a_{1010}+b_{0110}\right)}\right),
$$

Subcase 2.7: If $Q_{2} \neq 0, \mu \lambda<0$ and $Q_{1}>0$ system (7) has the simple zero

$$
\left(r_{7}, z_{7}\right)=\left(2 \sqrt{\frac{\omega\left(-6 Q_{2}-2 \sqrt{9 Q_{2}^{2}-12 Q_{1} \lambda \mu}\right)}{Q_{1}}}, \frac{B_{1} r_{7}^{2}+16 \lambda \omega}{8 \omega r_{7}\left(a_{1010}+b_{0110}\right)}\right),
$$

for $i=6,7$ the Jacobian determinant is $\operatorname{det}\left(D g_{2}\left(r_{i}, z_{i}\right)\right)=-\frac{64 \pi^{2} \lambda \mu}{\omega^{2}}-\frac{2 \pi^{2} Q_{2} r_{i}^{2}}{\omega^{3}} \neq 0$.

The result follows by applying Theorem 2 for each $a_{1}^{*}=\left(r_{i}, z_{i}\right)$ with $i=0,1, \ldots, 7$. This completes the proof of Theorem 1. 


\section{Applications}

The existence of differential systems with only zero, one or fewer than $n$ equilibrium points generating $n-$ scroll chaotic attractors is an important open problem whose solution is not easy. For more information about $n-$ scroll chaotic attractors see Lü \& Chen [2006]. This type of system has several real word applications, for instance in engineering and secure communication. Elhadj \& Sprott [2013] have shown that the simplest family of systems displaying $n$-scroll chaotic attractors is given by the quadratic polynomial differential system

$$
\begin{aligned}
& \dot{x}=a_{1} x+a_{2} y+a_{3} z+a_{9} y z, \\
& \dot{y}=b_{1} x+b_{2} y+b_{3} z+b_{8} x z+b_{9} y z, \\
& \dot{z}=c_{1} x+c_{2} y+c_{3} z+c_{7} x y+c_{8} x z+c_{9} y z .
\end{aligned}
$$

Using Theorem 1 we can find conditions in order that the differential system (10) has two periodic orbits.

Theorem 3. Consider system (10) with the coefficients

$$
\begin{aligned}
& a_{1}=\varepsilon^{2}, \quad a_{2}=\frac{\varepsilon(\varepsilon-\mu) k_{1}}{k_{3}}, \quad a_{3}=-k_{1}, \\
& a_{9}=-k_{2}, \quad b_{1}=\frac{\varepsilon(\varepsilon-\mu) k_{3}}{k_{1}}, \quad b_{2}=\varepsilon^{2}, \\
& b_{3}=-k_{3}, \quad b_{8}=\varepsilon^{2}, \quad b_{9}=-\frac{k_{2}\left(3239 k_{1}^{2}+5616 k_{3}^{2}\right)}{4992 k_{1} k_{3}}, \\
& c_{1}=\frac{\varepsilon^{4}+\omega^{2}}{k_{1}}, \quad c_{2}=\frac{\varepsilon^{2}(\varepsilon+25 \mu)(3 \varepsilon+25 \mu)}{k_{3}}, \quad c_{3}=-\varepsilon(2 \varepsilon+51 \mu), \\
& c_{7}=-\frac{79 \omega^{2} k_{2}}{24 k_{3}^{2}}, \quad c_{8}=\frac{k_{1} k_{4}+k_{3} k_{5}}{k_{1}}, \quad c_{9}=-k_{5} .
\end{aligned}
$$

Assume that $\omega>0, \mu>0$ and $k_{i}>0$ for all $i=1, \ldots, 5$. Then for $\varepsilon>0$ sufficiently small system (10) has two periodic orbits bifurcating from the zero-Hopf equilibrium point localized at the origin.

Furthermore we also shall use Theorem 1 for proving the existence of $\varepsilon$-families of periodic orbits in the following two differential systems

$$
\begin{aligned}
& \dot{x}=a(y-x)+d x z, \\
& \dot{y}=\rho x-x z+f y, \\
& \dot{z}=-e x^{2}+x y+c z,
\end{aligned}
$$

$$
\begin{aligned}
& \dot{x}=a(y-x)+d x z, \\
& \dot{y}=\left(f_{0}-a\right) x-x z+f_{1} y, \\
& \dot{z}=-e x^{2}+x y+c z+m,
\end{aligned}
$$

System (11) was derived from the classical Lorenz system by Li [2008]. This system exhibits a threescroll chaotic attractor, with two scrolls symmetric with respect to the $z$-axis as in the Lorenz attractor, and the third scroll is around the $z$-axis. System (12) were provided by Pan et al. [2010] with $f_{0}=f_{1}$. It was derived from the Chen system and also presents a three-scroll chaotic attractor. The authors show that the parameter $m$ works as a control parameter that can dramatically change the dynamics of the system. Theorem 5 will reveal that the parameter $m$ is also important for the existence of periodic orbits in system (12).

In the next results we used Theorem 1 to provide sufficient conditions for the existence of periodic orbits in systems (11) and (12) respectively.

Theorem 4. Let $c=\varepsilon \mu, f=a-2 \varepsilon \lambda, \rho=-\left(\omega^{2}+(a-\varepsilon \lambda)^{2}\right) / a$ and $e=1+\varepsilon \lambda / a$. If $a d \mu(\mu-4 \lambda) \lambda>0$ then system (11) has a family of periodic solutions bifurcating from the zero-Hopf equilibrium localized at the origin. 
Theorem 5. Let $m=\varepsilon m_{1}, c=-\varepsilon \mu, d=\left(a-f_{1}-2 \varepsilon \lambda\right) \mu / m_{1}$ and $f_{0}=\left(m_{1} a+a^{2} \mu-\left(f_{1}+\varepsilon \lambda\right)^{2} \mu-\mu \omega^{2}\right) /(a \mu)$. If $a\left(f_{1}-a\right) m_{1} \lambda(8 \lambda-\mu)<0$, then system (12) has a family of periodic solutions bifurcating from the zeroHopf equilibrium $p_{0}=\left(0,0, \frac{m_{1}}{\mu}\right)$.

\subsection{Proofs of Theorems 3, 4 and 5}

Proof. [Proof of Theorem 3] Using the linear change of variables

$$
\begin{aligned}
U & =\frac{z k_{1} \varepsilon^{2}\left(677 \mu^{2}+\varepsilon^{2}+50 \mu \varepsilon\right)}{k_{3}\left(\varepsilon^{4}+50 \mu \varepsilon^{3}-52 \mu^{2} \varepsilon^{2}-\omega^{2}\right)}+x, \\
V & =\frac{x k_{3}}{k_{1}}+z, \\
W & =\frac{x \varepsilon(25 \mu+2 \varepsilon)+y \omega}{k_{3}}+\frac{z \varepsilon(\varepsilon-\mu)\left(2 \varepsilon^{4}+100 \mu \varepsilon^{3}+625 \mu^{2} \varepsilon^{2}-\omega^{2}\right)}{k_{3}\left(\varepsilon^{4}+50 \mu \varepsilon^{3}-52 \mu^{2} \varepsilon^{2}-\omega^{2}\right)},
\end{aligned}
$$

system (10) becomes

$$
\begin{aligned}
& \dot{U}=-\varepsilon 26 \mu U-\omega V+\sum_{i+j+k=2} a_{i j k}(\varepsilon) U^{i} V^{j} W^{k}, \\
& \dot{V}=\omega U-\varepsilon 26 \mu V+\sum_{i+j+k=2} b_{i j k}(\varepsilon) U^{i} V^{j} W^{k}, \\
& \dot{W}=\varepsilon \mu W+\sum_{i+j+k=2} c_{i j k}(\varepsilon) U^{i} V^{j} W^{k} .
\end{aligned}
$$

Where the functions $a_{i j k}(\varepsilon), b_{i j k}(\varepsilon)$ and $c_{i j k}(\varepsilon)$ are defined in the appendix. We note that when $\varepsilon=0$ the origin of system (13) is a non-isolated zero-Hopf equilibrium point. Since

$$
c_{1100}=-\frac{\omega\left(3239 k_{1}^{2} k_{2}+624 k_{2} k_{3}^{2}\right)}{4992 k_{1}^{3}} \neq 0,
$$

system (13) does not satisfy the hypothesis $\left(H_{1}\right)$ of [Llibre \& Xiao, 2014, Theorem 2.4]. However, we have $a_{0020}=b_{0020}=c_{0020}=0$ and $c_{2000}=-c_{0200}=0$ thus we can apply Theorem 1. From the coefficients of system (13) we have $\lambda=-26 \mu$ and

$$
\begin{aligned}
a_{1010} & =0 \\
b_{0110} & =-k_{5}, \\
Q_{1} & =-\frac{395 k_{2}^{2} k_{4} k_{5} \omega^{2}\left(3239 k_{1}^{2}+624 k_{3}^{2}\right)}{1038336 k_{1}^{3} k_{3}}, \\
Q_{2} & =\frac{k_{2} \mu \omega\left(32864 k_{1}^{3} k_{4}+16195 k_{1}^{2} k_{3} k_{5}+3120 k_{3}^{3} k_{5}\right)}{4992 k_{1}^{3} k_{3}}, \\
3 Q_{2}^{2}-4 \lambda \mu Q_{1} & =\frac{32448 k_{3}^{2} Q_{1}^{2} \mu^{2}}{6241 k_{2}^{2} k_{4}^{2} \omega^{2}}+\frac{6241 k_{2}^{2} k_{4}^{2} \omega^{2} \mu^{2}}{192 k_{3}^{2}} .
\end{aligned}
$$

By equations (14) we have that $a_{1010}-b_{0110} \neq 0$ and $3 Q_{2}^{2}-4 \mu \lambda Q_{1}>0$. Furthermore if $\mu>0$ we have $Q_{2}>0, \lambda \mu=-26 \mu^{2}<0$ and $3 Q_{2}^{2} /(4 \lambda \mu)<Q_{1}<0$, then system (10) satisfies statement $(h)$ of Theorem 1. Then the theorem is proved.

Proof. [Proof of Theorem 4] Under the hypothesis of Theorem 4 system (11) has three equilibrium points. Mainly, the origin and $p_{ \pm}=\left( \pm x_{\varepsilon}, \pm y_{\varepsilon}, z_{\varepsilon}\right)$ where

$$
x_{\varepsilon}=\sqrt{\frac{a \mu \varepsilon\left(\omega^{2}+\varepsilon^{2} \lambda^{2}\right)}{d \omega-\lambda \varepsilon(a+a d+d \varepsilon \lambda)}},
$$




$$
\begin{aligned}
& y_{\varepsilon}=x_{\varepsilon} \frac{a^{2}(d+1)+2 a d \lambda \varepsilon+d\left(\omega^{2}+\lambda^{2} \varepsilon^{2}\right)}{a(a d+a+2 d \lambda \varepsilon)}, \\
& z_{\varepsilon}=\frac{\omega^{2}+\lambda^{2} \varepsilon^{2}}{a d+a+2 d \lambda \varepsilon} .
\end{aligned}
$$

The eigenvalues at the origin are $\varepsilon \mu, \varepsilon \lambda-i \omega$ and $\varepsilon \lambda+i \omega$. Applying the change of variables $(x, y, z)=$ $(U,(U a-U \varepsilon \lambda-V \omega) / \omega, W)$ the differential system (11) becomes

$$
\begin{aligned}
\dot{U} & =\varepsilon \lambda U-\omega V+a_{101}(\varepsilon) U W, \\
\dot{V} & =\omega U+\varepsilon \lambda V+b_{101}(\varepsilon) U W, \\
\dot{W} & =\varepsilon \mu W+c_{110}(\varepsilon) U V,
\end{aligned}
$$

where $a_{101}(\varepsilon)=d, b_{101}(\varepsilon)=\frac{a+a d+\varepsilon d \lambda}{\omega}$ and $c_{110}(\varepsilon)=\frac{\omega}{a}$.

We note that when $\varepsilon=0$ the origin of system (15) is a non-isolated zero-Hopf equilibrium point. Consequently we have $a_{1010}-b_{0110}=d, Q_{1}=0$ and $Q_{2}=d \omega(\mu-4 \lambda) / a$. Thus by hypotheses system (15) satisfies the conditions $(c)$ of Theorem 1 . Hence the theorem is proved.

Proof. [Proof of Theorem 5] The point $p_{0}=\left(0,0, m_{1} / \mu\right)$ is an equilibrium of system (12). We translate $p_{0}$ to the origin doing the following change of variables

$$
\begin{aligned}
(x, y, z) & =\left(U, \frac{f_{1} U+\varepsilon \lambda U-\omega V}{a}, W+\frac{m_{1}}{\mu}\right) . \\
\dot{U} & =\varepsilon \lambda U-\omega V+a_{101}(\varepsilon) U W \\
\dot{V} & =\omega U+\varepsilon \lambda V+b_{101}(\varepsilon) U W \\
\dot{W} & =\varepsilon \mu W+c_{200}(\varepsilon) U^{2}+c_{110}(\varepsilon) U V .
\end{aligned}
$$

Where the functions (2) vanishing except for

$$
\begin{aligned}
a_{101}(\varepsilon) & =\frac{\left(a-f_{1}-2 \varepsilon \lambda\right)}{m_{1}}, & b_{101}(\varepsilon) & =\frac{a m_{1}+\left(a-f_{1}-2 \varepsilon \lambda\right)\left(f_{1}+\varepsilon \lambda\right) \mu}{m_{1} \omega}, \\
c_{200}(\varepsilon) & =\varepsilon \frac{\lambda}{a}, & c_{110}(\varepsilon) & =\frac{\omega}{a} .
\end{aligned}
$$

We note that when $\varepsilon=0$ the origin of system (16) is a non-isolated zero-Hopf equilibrium point. Thus we have

$$
a_{1010}-b_{0110}=\frac{\mu\left(a-f_{1}\right)}{m_{1}}, \quad Q_{1}=0, \quad Q_{2}=\frac{\left(f_{1}-a\right)(8 \lambda-\mu) \mu \omega}{a m_{1}} .
$$

Consequently system (15) satisfies the conditions $(c)$ of Theorem 1 since we have by hypothesis that

$$
\lambda \mu Q_{2}=\frac{\lambda\left(f_{1}-a\right)(8 \lambda-\mu) \mu^{2} \omega}{a m_{1}}<0
$$

This concludes the proof of the theorem.

\section{Appendix}

Here we write the coefficients of system (13).

$$
a_{200}(\varepsilon)=\frac{\varepsilon(25 \mu+2 \varepsilon)\left(k_{1}^{2} \varepsilon^{2}\left(4992 \varepsilon^{2}-3239 k_{2}\right)\left(677 \mu^{2}+\varepsilon^{2}+50 \mu \varepsilon\right)\right)}{4992 k_{1}^{2} k_{3}\left(\omega^{2}+729 \mu^{2} \varepsilon^{2}\right)}
$$




$$
\begin{aligned}
& a_{020}(\varepsilon)=0, \\
& a_{002}(\varepsilon)=\frac{\varepsilon(\varepsilon-\mu)\left(-\omega^{2}+2 \varepsilon^{4}+100 \mu \varepsilon^{3}+625 \mu^{2} \varepsilon^{2}\right)}{4992 k_{3}^{3}\left(-\omega^{2}+\varepsilon^{4}+50 \mu \varepsilon^{3}-52 \mu^{2} \varepsilon^{2}\right)^{2}\left(\omega^{2}+729 \mu^{2} \varepsilon^{2}\right)} \\
& \times\left(k _ { 1 } ^ { 2 } \varepsilon ^ { 2 } ( 6 7 7 \mu ^ { 2 } + \varepsilon ^ { 2 } + 5 0 \mu \varepsilon ) \left(4992 \varepsilon^{4}\left(677 \mu^{2}+\varepsilon^{2}+50 \mu \varepsilon\right)\right.\right. \\
& \left.-3239 k_{2}\left(-\omega^{2}+\varepsilon^{4}+50 \mu \varepsilon^{3}-52 \mu^{2} \varepsilon^{2}\right)\right)-624 k_{2} k_{3}^{2}\left(-8 \omega^{4}\right. \\
& +\varepsilon^{8}+100 \mu \varepsilon^{7}+8957 \mu^{2} \varepsilon^{6}+322850 \mu^{3} \varepsilon^{5}+\varepsilon^{4}\left(7 \omega^{2}-338468 \mu^{4}\right) \\
& \left.\left.+350 \mu \omega^{2} \varepsilon^{3}-6925 \mu^{2} \omega^{2} \varepsilon^{2}\right)\right) \text {, } \\
& a_{110}(\varepsilon)=-\frac{\omega}{4992 k_{1}^{2} k_{3}\left(\omega^{2}+729 \mu^{2} \varepsilon^{2}\right)}\left(k_{1}^{2} \varepsilon^{2}\left(3239 k_{2}-4992 \varepsilon^{2}\right)\right. \\
& \left.\left(677 \mu^{2}+\varepsilon^{2}+50 \mu \varepsilon\right)+624 k_{2} k_{3}^{2}\left(8 \omega^{2}+\varepsilon^{4}+50 \mu \varepsilon^{3}+6509 \mu^{2} \varepsilon^{2}\right)\right), \\
& a_{101}(\varepsilon)=\frac{\varepsilon}{4992 k_{1} k_{3}^{2}\left(-\omega^{2}+\varepsilon^{4}+50 \mu \varepsilon^{3}-52 \mu^{2} \varepsilon^{2}\right)\left(\omega^{2}+729 \mu^{2} \varepsilon^{2}\right)} \\
& \left(k _ { 1 } ^ { 2 } \varepsilon ^ { 2 } ( 6 7 7 \mu ^ { 2 } + \varepsilon ^ { 2 } + 5 0 \mu \varepsilon ) \left(3 2 3 9 k _ { 2 } \left(3 \omega^{2}(8 \mu+\varepsilon)\right.\right.\right. \\
& \left.-\varepsilon^{2}\left(-1925 \mu^{3}+4 \varepsilon^{3}+223 \mu \varepsilon^{2}+1671 \mu^{2} \varepsilon\right)\right)+4992\left(\omega^{2} \varepsilon^{2}(\mu-\varepsilon)\right. \\
& \left.\left.+\varepsilon^{4}\left(16300 \mu^{3}+4 \varepsilon^{3}+223 \mu \varepsilon^{2}+3129 \mu^{2} \varepsilon\right)\right)\right)-624 k_{2} k_{3}^{2}\left(8 \omega^{2}\right. \\
& \left.+\varepsilon^{4}+50 \mu \varepsilon^{3}+6509 \mu^{2} \varepsilon^{2}\right)\left(\varepsilon^{2}\left(-1925 \mu^{3}+4 \varepsilon^{3}+223 \mu \varepsilon^{2}+1671 \mu^{2} \varepsilon\right)\right. \\
& \left.\left.-3 \omega^{2}(8 \mu+\varepsilon)\right)\right) \text {, } \\
& a_{011}(\varepsilon)=\frac{\omega}{4992 k_{1}}\left(\frac{\varepsilon^{2}\left(677 \mu^{2}+\varepsilon^{2}+50 \mu \varepsilon\right)\left(k_{1}^{2}\left(4992 \varepsilon^{2}-3239 k_{2}\right)-624 k_{2} k_{3}^{2}\right)}{k_{3}^{2}\left(\omega^{2}+729 \mu^{2} \varepsilon^{2}\right)}\right. \\
& \left.+\frac{4992 k_{1}^{2} \varepsilon^{4}\left(677 \mu^{2}+\varepsilon^{2}+50 \mu \varepsilon\right)}{k_{3}^{2}\left(-\omega^{2}+\varepsilon^{4}+50 \mu \varepsilon^{3}-52 \mu^{2} \varepsilon^{2}\right)}-4992 k_{2}\right) \\
& b_{200}(\varepsilon)=\frac{1}{4992 k_{1}^{2} k_{3} \omega\left(729 \varepsilon^{2} \mu^{2}+\omega^{2}\right)}\left(k _ { 1 } ^ { 2 } \left(7 9 k _ { 2 } \left(-208 \omega^{4}+\omega^{2} \varepsilon^{2}\left(-152657 \mu^{2}\right.\right.\right.\right. \\
& \left.\left.+82 \varepsilon^{2}+943 \mu \varepsilon\right)+1107 \mu \varepsilon^{4}(25 \mu+2 \varepsilon)\left(650 \mu^{2}+\varepsilon^{2}+77 \mu \varepsilon\right)\right) \\
& +4992 \varepsilon(25 \mu+2 \varepsilon)\left(\omega^{2}\left(k_{3} k_{4}+\varepsilon^{3}(\mu-\varepsilon)\right)-27 \mu \varepsilon^{2}\left(-27 k_{3} k_{4} \mu+\varepsilon^{5}\right.\right. \\
& \left.\left.\left.+77 \mu \varepsilon^{4}+650 \mu^{2} \varepsilon^{3}\right)\right)\right)+624 k_{2} k_{3}^{2} \varepsilon^{2}(25 \mu+2 \varepsilon)\left(2 7 \mu \varepsilon ^ { 2 } \left(6050 \mu^{2}+\varepsilon^{2}\right.\right. \\
& \left.\left.+509 \mu \varepsilon)+\omega^{2}(199 \mu+17 \varepsilon)\right)\right) \text {, } \\
& b_{020}(\varepsilon)=0, \\
& b_{002}(\varepsilon)=\frac{\varepsilon}{4992 k_{3}^{3}\left(-\omega^{2}+\varepsilon^{4}+50 \mu \varepsilon^{3}-52 \mu^{2} \varepsilon^{2}\right)^{2}\left(\omega^{3}+729 \mu^{2} \omega \varepsilon^{2}\right)} \\
& \left(k _ { 1 } ^ { 2 } \varepsilon \left(7 9 k _ { 2 } ( - \omega ^ { 2 } + \varepsilon ^ { 4 } + 5 0 \mu \varepsilon ^ { 3 } - 5 2 \mu ^ { 2 } \varepsilon ^ { 2 } ) \left(-\omega^{4}\left(140857 \mu^{2}+249 \varepsilon^{2}\right.\right.\right.\right. \\
& +10318 \mu \varepsilon)+1107 \mu \varepsilon^{4}(\varepsilon-\mu)\left(625 \mu^{2}+2 \varepsilon^{2}+100 \mu \varepsilon\right)\left(650 \mu^{2}+\varepsilon^{2}\right. \\
& +77 \mu \varepsilon)+\omega^{2} \varepsilon^{2}\left(-101909689 \mu^{4}+82 \varepsilon^{4}+2829 \mu \varepsilon^{3}-218257 \mu^{2} \varepsilon^{2}\right. \\
& \left.\left.-8263061 \mu^{3} \varepsilon\right)\right)-4992 \varepsilon(\varepsilon-\mu)\left(677 \mu^{2}+\varepsilon^{2}+50 \mu \varepsilon\right)\left(-\omega^{2}+2 \varepsilon^{4}\right. \\
& \left.+100 \mu \varepsilon^{3}+625 \mu^{2} \varepsilon^{2}\right)\left(\omega^{2}\left(-k_{3} k_{4}+\varepsilon^{4}-\mu \varepsilon^{3}\right)+27 \mu \varepsilon^{2}\left(-27 k_{3} k_{4} \mu\right.\right. \\
& \left.\left.\left.+\varepsilon^{5}+77 \mu \varepsilon^{4}+650 \mu^{2} \varepsilon^{3}\right)\right)\right)+4992 k_{1} k_{3}^{2} k_{5}(\varepsilon-\mu)\left(-\omega^{2}+2 \varepsilon^{4}\right.
\end{aligned}
$$




$$
\begin{aligned}
& \left.+100 \mu \varepsilon^{3}+625 \mu^{2} \varepsilon^{2}\right)\left(\omega^{2}+729 \mu^{2} \varepsilon^{2}\right)^{2}+624 k_{2} k_{3}^{2} \varepsilon(\varepsilon-\mu)\left(-\omega^{2}\right. \\
& \left.+2 \varepsilon^{4}+100 \mu \varepsilon^{3}+625 \mu^{2} \varepsilon^{2}\right)\left(\varepsilon^{4}+50 \mu \varepsilon^{3}-52 \mu^{2} \varepsilon^{2}-\omega^{2}\right) \\
& \left.\left.\left(27 \mu \varepsilon^{2}\left(6050 \mu^{2}+\varepsilon^{2}+509 \mu \varepsilon\right)+\omega^{2}(199 \mu+17 \varepsilon)\right)\right)\right) \text {, } \\
& b_{110}(\varepsilon)=\frac{k_{1}^{2}}{4992 k_{1}^{2} k_{3}\left(\omega^{2}+729 \mu^{2} \varepsilon^{2}\right)}\left(2 7 \mu \varepsilon ^ { 2 } \left(\varepsilon ( 3 2 3 9 k _ { 2 } - 4 9 9 2 \varepsilon ^ { 2 } ) \left(650 \mu^{2}+\varepsilon^{2}\right.\right.\right. \\
& \left.+77 \mu \varepsilon)+134784 k_{3} k_{4} \mu\right)+\omega^{2}\left(\varepsilon\left(3239 k_{2}-4992 \varepsilon^{2}\right)(\varepsilon-\mu)\right. \\
& \left.\left.+4992 k_{3} k_{4}\right)\right)+624 k_{2} k_{3}^{2} \varepsilon\left(27 \mu \varepsilon^{2}\left(6050 \mu^{2}+\varepsilon^{2}+509 \mu \varepsilon\right)\right. \\
& \left.+\omega^{2}(199 \mu+17 \varepsilon)\right) \\
& b_{101}(\varepsilon)=\frac{k_{1}^{2}}{4992 k_{1} k_{3}^{2} \omega\left(\omega^{2}+729 \mu^{2} \varepsilon^{2}\right)\left(\omega^{2}-\varepsilon^{2}\left(-52 \mu^{2}+\varepsilon^{2}+50 \mu \varepsilon\right)\right)} \\
& \left(7 9 k _ { 2 } \left(-208 \omega^{6}+\omega^{4} \varepsilon^{2}\left(-22616 \mu^{2}+539 \varepsilon^{2}+21661 \mu \varepsilon\right)\right.\right. \\
& -1107 \mu \varepsilon^{6}\left(650 \mu^{2}+\varepsilon^{2}+77 \mu \varepsilon\right)\left(-1925 \mu^{3}+4 \varepsilon^{3}+223 \mu \varepsilon^{2}\right. \\
& \left.+1671 \mu^{2} \varepsilon\right)+\omega^{2} \varepsilon^{4}\left(111960275 \mu^{4}-164 \varepsilon^{4}-5658 \mu \varepsilon^{3}+526181 \mu^{2} \varepsilon^{2}\right. \\
& \left.\left.+19515022 \mu^{3} \varepsilon\right)\right)+4992 \varepsilon\left(\mu \omega^{2}+4 \varepsilon^{5}+223 \mu \varepsilon^{4}+3129 \mu^{2} \varepsilon^{3}\right. \\
& \left.+16300 \mu^{3} \varepsilon^{2}-\omega^{2} \varepsilon\right)\left(\omega^{2}\left(-k_{3} k_{4}+\varepsilon^{4}-\mu \varepsilon^{3}\right)+27 \mu \varepsilon^{2}\left(-27 k_{3} k_{4} \mu\right.\right. \\
& \left.\left.\left.+\varepsilon^{5}+77 \mu \varepsilon^{4}+650 \mu^{2} \varepsilon^{3}\right)\right)\right)-4992 k_{1} k_{3}^{2} k_{5} \varepsilon(25 \mu+2 \varepsilon) \\
& \left(\omega^{2}+729 \mu^{2} \varepsilon^{2}\right)^{2}-624 k_{2} k_{3}^{2} \varepsilon^{2}\left(\varepsilon ^ { 2 } \left(-1925 \mu^{3}+4 \varepsilon^{3}+223 \mu \varepsilon^{2}\right.\right. \\
& \left.\left.+1671 \mu^{2} \varepsilon\right)-3 \omega^{2}(8 \mu+\varepsilon)\right)\left(27 \mu \varepsilon^{2}\left(6050 \mu^{2}+\varepsilon^{2}+509 \mu \varepsilon\right)\right. \\
& \left.+\omega^{2}(199 \mu+17 \varepsilon)\right) \text {, } \\
& b_{011}(\varepsilon)=0, \\
& c_{200}(\varepsilon)=\frac{\varepsilon(25 \mu+2 \varepsilon)}{4992 k_{1}^{3}\left(\omega^{2}+729 \mu^{2} \varepsilon^{2}\right)}\left(-\omega^{2}+\varepsilon^{4}+50 \mu \varepsilon^{3}-52 \mu^{2} \varepsilon^{2}\right) \\
& \left(k_{1}^{2}\left(3239 k_{2}-4992 \varepsilon^{2}\right)+624 k_{2} k_{3}^{2}\right), \\
& c_{020}(\varepsilon)=0 \text {, } \\
& c_{002}(\varepsilon)=-\frac{\varepsilon(\varepsilon-\mu)\left(-\omega^{2}+2 \varepsilon^{4}+100 \mu \varepsilon^{3}+625 \mu^{2} \varepsilon^{2}\right)}{4992 k_{1} k_{3}^{2}\left(-\omega^{2}+\varepsilon^{4}+50 \mu \varepsilon^{3}-52 \mu^{2} \varepsilon^{2}\right)\left(\omega^{2}+729 \mu^{2} \varepsilon^{2}\right)} \\
& \left(k _ { 1 } ^ { 2 } \left(4992 \varepsilon^{4}\left(677 \mu^{2}+\varepsilon^{2}+50 \mu \varepsilon\right)-3239 k_{2}\left(-\omega^{2}+\varepsilon^{4}+50 \mu \varepsilon^{3}\right.\right.\right. \\
& \left.\left.\left.-52 \mu^{2} \varepsilon^{2}\right)\right)+624 k_{2} k_{3}^{2}\left(\omega^{2}-\varepsilon^{2}\left(-52 \mu^{2}+\varepsilon^{2}+50 \mu \varepsilon\right)\right)\right), \\
& c_{110}(\varepsilon)=\frac{\omega\left(-\omega^{2}+\varepsilon^{4}+50 \mu \varepsilon^{3}-52 \mu^{2} \varepsilon^{2}\right)}{4992 k_{1}^{3}\left(\omega^{2}+729 \mu^{2} \varepsilon^{2}\right)} \\
& \left(k_{1}^{2}\left(3239 k_{2}-4992 \varepsilon^{2}\right)+624 k_{2} k_{3}^{2}\right) \text {, } \\
& c_{101}(\varepsilon)=\frac{1}{4992 k_{1}^{2} k_{3}\left(\omega^{2}+729 \mu^{2} \varepsilon^{2}\right)}\left(k _ { 1 } ^ { 2 } \varepsilon \left(3 2 3 9 k _ { 2 } \left(\varepsilon ^ { 2 } \left(-1925 \mu^{3}\right.\right.\right.\right. \\
& \left.\left.+4 \varepsilon^{3}+223 \mu \varepsilon^{2}+1671 \mu^{2} \varepsilon\right)-3 \omega^{2}(8 \mu+\varepsilon)\right)-4992 \varepsilon^{2}\left(\mu \omega^{2}+4 \varepsilon^{5}\right. \\
& \left.\left.+223 \mu \varepsilon^{4}+3129 \mu^{2} \varepsilon^{3}+16300 \mu^{3} \varepsilon^{2}-\omega^{2} \varepsilon\right)\right) \\
& +624 k_{2} k_{3}^{2} \varepsilon\left(\varepsilon^{2}\left(-1925 \mu^{3}+4 \varepsilon^{3}+223 \mu \varepsilon^{2}+1671 \mu^{2} \varepsilon\right)\right. \\
& \left.\left.-3 \omega^{2}(8 \mu+\varepsilon)\right)\right) \text {, } \\
& c_{011}(\varepsilon)=-\frac{\omega}{4992 k_{1}^{2} k_{3}\left(\omega^{2}+729 \mu^{2} \varepsilon^{2}\right)}\left(k _ { 1 } ^ { 2 } \left(4992 \varepsilon^{4}\left(677 \mu^{2}+\varepsilon^{2}+50 \mu \varepsilon\right)\right.\right.
\end{aligned}
$$




$$
\begin{aligned}
& \left.-3239 k_{2}\left(\varepsilon^{4}+50 \mu \varepsilon^{3}-52 \mu^{2} \varepsilon^{2}-\omega^{2}\right)\right) \\
& \left.-624 k_{2} k_{3}^{2}\left(\varepsilon^{4}+50 \mu \varepsilon^{3}-52 \mu^{2} \varepsilon^{2}-\omega^{2}\right)\right) .
\end{aligned}
$$

\section{Acknowledgements}

We thank to the reviewer his comments and suggestions which help us to improve the presentation of this paper.

The first author is partially supported by CNPq 248501/2013-5. The second author is partially supported by a FEDER-MINECO grant MTM2016-77278-P, a MINECO grant MTM2013-40998-P, and an AGAUR grant number 2014SGR-568.

\section{References}

Cândido, M. R., Llibre, J. \& Novaes, D. D. [2016] "Persistence of periodic solutions for higher order perturbed differential systems via lyapunov-schmidt reduction," arXiv preprint arXiv:1611.04807 .

Coll, B., Gasull, A. \& Prohens, R. [2012] "Periodic orbits for perturbed non-autonomous differential equations," Bulletin des Sciences Mathématiques 136, 803-819.

Elhadj, Z. \& Sprott, J. C. [2013] "Simplest 3d continuous-time quadratic systems as candidates for generating multiscroll chaotic attractors," International Journal of Bifurcation and Chaos 23, 1350120.

Giné, J., Llibre, J., Wu, K. \& Zhang, X. [2016] "Averaging methods of arbitrary order, periodic solutions and integrability," Journal of Differential Equations 260, 4130-4156.

Guckenheimer, J. [1981] "On a codimension two bifurcation," Dynamical Systems and Turbulence, Warwick $1980,99-142$.

Guckenheimer, J. \& Holmes, P. J. [2013] Nonlinear oscillations, dynamical systems, and bifurcations of vector fields, Vol. 42 (Springer Science \& Business Media).

Kuznetsov, Y. A. [2013] Elements of applied bifurcation theory, Vol. 112 (Springer Science \& Business Media).

Li, D. [2008] "A three-scroll chaotic attractor," Physics Letters A 372, 387-393.

Llibre, J., Novaes, D. D. \& Teixeira, M. A. [2014] "Higher order averaging theory for finding periodic solutions via brouwer degree," Nonlinearity 27, 563.

Llibre, J. \& Xiao, D. [2014] "Limit cycles bifurcating from a non-isolated zero-hopf equilibrium of threedimensional differential systems," Proceedings of the American Mathematical Society 142, 2047-2062.

Lü, J. \& Chen, G. [2006] "Generating multiscroll chaotic attractors: theories, methods and applications," International Journal of Bifurcation and Chaos 16, 775-858.

Murdock, J., Sanders, J. \& Verhulst, F. [2007] "Averaging methods in nonlinear dynamical systems," Appl. Math. Sci $\mathbf{5 9 .}$

Pan, L., Zhou, W. \& Fang, J. [2010] "On dynamics analysis of a novel three-scroll chaotic attractor," Journal of the Franklin Institute 347, 508-522.

Scheurle, J. \& Marsden, J. [1984] "Bifurcation to quasi-periodic tori in the interaction of steady state and hopf bifurcations," SIAM journal on mathematical analysis 15, 1055-1074.

Tian, H. \& Han, M. [2017] "Bifurcation of periodic orbits by perturbing high-dimensional piecewise smooth integrable systems," Journal of Differential Equations 263, 7448-7474. 